\title{
Conhecimento e práticas sobre raiva junto às Unidades Básicas de Saúde no Brasil
}

\author{
Knowledge and practices about rabies at Basic Health Units in Brazil \\ Conocimientos y prácticas sobre la rabia en las Unidades Básicas de Salud de Brasil
}

Recebido: 06/01/2022 | Revisado: 11/01/2022 | Aceito: 12/01/2022 | Publicado: 14/01/2022

\author{
Vinicius de Lima Lovadini \\ ORCID: https://orcid.org/0000-0001-9066-2160 \\ Universidade Estadual Paulista, Brasil \\ E-mail: viniciuslovadini@usp.br \\ Laura Luisa Gonçalves Lorena \\ ORCID: https://orcid.org/0000-0003-1358-2831 \\ Universidade Estadual Paulista, Brasil \\ E-mail: lauraglorena@ @otmail.com \\ Jaqueline da Silva Lacerda \\ ORCID: https://orcid.org/0000-0003-4315-394X \\ Universidade Estadual Paulista, Brasil \\ E-mail: já-lacerda@hotmail.com \\ Matheus Janeck Araújo \\ ORCID: https://orcid.org/0000-0002-6588-4578 \\ Universidade Estadual Paulista, Brasil \\ E-mail: mathjaneck@hotmail.com \\ Márcia Marinho \\ ORCID: https://orcid.org/0000-0003-2177-6214 \\ Universidade Estadual Paulista, Brasil \\ E-mail: marcia.marinho@unesp.br
}

\begin{abstract}
Resumo
Objetivo: mapear o padrão da distribuição espacial sobre o conhecimento e práticas de vacinação acerca da zoonose Raiva na população atendida nas Unidades Básicas de Saúde do município de Birigui, São Paulo, Brasil. Método: estudo epidemiológico observacional analítico, não experimental, com delineamento transversal. Foram aplicados 400 questionários entre janeiro e julho de 2019 para os participantes das 10 Unidades Básicas de Saúde do município de Birigui de forma randomizada, nas análises espaciais foi utilizado o Estimador de Densidades de Kernel. Resultados: 74,25\% (n=297) dos participantes são do sexo feminino, 44\% (n=176) alocavam-se entre 31-50 anos de idade, e 67\% $(\mathrm{n}=268)$ da população apontaram corretamente os animais transmissores da Raiva. Identificou-se clusters com maiores densidades de conhecimentos e práticas nas Unidades Básicas de Saúde bem como os mais vulneráveis. Conclusão: As análises espaciais contribuíram para uma avaliação mais específica do perfil do conhecimento dos munícipes sobre a Raiva.
\end{abstract}

Palavras-chave: Raiva; Centros de saúde; Meio ambiente e saúde pública; Análise espacial; Inquéritos epidemiológicos.

\begin{abstract}
Objective: to map the pattern of spatial distribution of knowledge and vaccination practices regarding zoonosis rabies in the population assisted in Basic Health Units in the municipality of Birigui, São Paulo, Brazil. Method: analytical, non-experimental observational epidemiological study with a cross-sectional design. 400 questionnaires were randomly applied between January and July 2019 to the participants of the 10 Basic Health Units in the city of Birigui. The Kernel Density Estimator was used in the spatial analysis. Results: $74.25 \%(\mathrm{n}=297)$ of the participants are female, $44 \%(n=176)$ were between $31-50$ years of age, and $67 \%(n=268)$ of the population correctly pointed the animals rabies transmitters. Clusters with higher densities of knowledge and practices in Basic Health Units were identified, as well as the most vulnerable. Conclusion: Spatial analyzes contributed to a more specific assessment of the profile of knowledge of residents about rabies.
\end{abstract}

Keywords: Rabies; Health centers; Environment and public health; Spatial analysis; Health surveys.

\section{Resumen}

Objetivo: mapear el patrón de distribución espacial del conocimiento y las prácticas de vacunación sobre zoonosis rabia en la población atendida en Unidades Básicas de Salud del municipio de Birigui, São Paulo, Brasil. Método: estudio epidemiológico observacional analítico no experimental con diseño transversal. Se aplicaron 400 cuestionarios aleatoriamente entre enero y julio de 2019 a los participantes de las 10 Unidades Básicas de Salud de la ciudad de Birigui, en el análisis espacial se utilizó el Estimador de Densidad Kernel. Resultados: el 74,25\% (n = 297) de los 
participantes son mujeres, el 44\% $(n=176)$ tenían entre 31 y 50 años y el $67 \%(n=268)$ de la población señaló correctamente los animales transmisores de rabia. Se identificaron los clusters con mayor densidad de conocimientos y prácticas en las Unidades Básicas de Salud, así como los más vulnerables. Conclusión: Los análisis espaciales contribuyeron a una evaluación más específica del perfil de conocimiento de los residentes sobre la rabia.

Palabras clave: Rabia; Centros de salud; Medio ambiente y salud pública; Análisis espacial; Encuestas epidemiológicas.

\section{Introdução}

Raiva é uma enfermidade infectocontagiosa geralmente fatal, caracterizada por sinais nervosos como agressividade, paresia e paralisia. É uma zoonose de etiologia viral, pertencente ao gênero Lyssavirus, da família Rhabdoviridae, classificado na ordem Mononegavirales (Ito et al., 2001). Atualmente, é considerada o maior problema de saúde pública pela Organização Mundial de Saúde (OMS) sendo responsável por um caso fatal a cada nove minutos, totalizando em 59.000 mortes humanas anualmente, sendo que $99 \%$ dos casos são transmitidos por mordeduras de cães, e $40 \%$ das vítimas são crianças. Embora seja uma enfermidade antiga, é insidiosa, negligenciada e pouco abordada pelos governos e comunidade internacional (WHO,2016; WHO, 2019).

A fim de reduzir esse cenário, a OMS, a Organização Mundial de Saúde Animal (OIE) e a Organização das Nações Unidas para Agricultura e Alimentação (FAO) estabeleceram a meta de eliminar a Raiva humana transmitida por cães em países endêmicos até 2030, estabelecendo um Plano de estratégias Globais. Essa cartilha resume a epidemiologia da Raiva, esforços globais para diagnosticar, controlar e eliminar a transmissão por animais terrestres e os mecanismos e fisiopatologia da infecção pelo Rhabdovirus (WHO, 2019; Vos et al., 2011).

No Brasil, como em alguns outros países, a Raiva é endêmica, apresentando uma epidemiologia particular representada pelos ciclos epidemiológicos, envolvendo o ciclo aéreo, transmitido por quirópteros; o silvestre terrestre, no qual os animais transmissores são os carnívoros silvestres e primatas não humanos; o rural, que engloba os animais de produção; e o urbano que é propagado principalmente pelo cão e gato. O ser humano por sua vez, pode ser infectado com o vírus proveniente de qualquer ciclo (Vos et al., 2011).

Entre as décadas de 1950 e 1960 os estados e municípios brasileiros expandiram suas atividades e regulamentações voltadas ao controle de zoonoses, em específico, da Raiva urbana, devido ao elevado número de casos de Raiva humana transmitida por cães. As medidas de controle adotadas foram imunização em massa de cães e gatos, apreensão e controle de animais errantes e campanhas de bloqueios em áreas de foco com grande circulação do vírus (Vos et al., 2011).

Na região noroeste do estado de São Paulo, que abrange a região de Araçatuba, foi registrada uma epidemia de Raiva canina no início de 1991 no município de Andradina e se estendeu aos municípios adjacentes, num raio de 26,4km por ano, afetando 10 municípios, e resultando em dois casos de Raiva humana em Araçatuba e Avanhandava nos anos de 1994 e 1997, respectivamente. Os cães representaram $87,2 \%$ do total de amostras positivas, sendo registrados ainda casos em felinos, equinos, bovinos e outras espécies (Queiroz et al., 2009).

No estado de São Paulo, a partir de 1994, ocorreu uma considerável queda no número de casos de Raiva canina, com um subsequente decréscimo da Raiva em humanos, transmitida pelo cão. No entanto, os ciclos selváticos estão se expandindo com um número crescente de diagnósticos em espécies como a raposa Cerdocyon thousand e um sagui comum Callythrix jacchus. No Brasil, morcegos vampiros também desempenham um papel principal na transmissão da Raiva (Ito et al., 2001). Consequentemente, a ocorrência do vírus da Raiva em morcegos hematófagos é refletida pela incidência da Raiva em bovinos (Gomes et al., 2009).

De acordo com o Instituto Pasteur, entre 2016 e 2020, no estado de São Paulo, foram diagnosticados 1479 casos de Raiva animal, desses, 657 ocorreram em bovinos, 530 em morcegos não hematófagos, 211 em equinos, 27 nos morcegos hematófagos, 23 em ovinos e caprinos, oito em felinos, seis em cães, seis em animais silvestres, quatro em muares, três em 
bubalinos e dois em asininos. E neste mesmo período, no município de Birigui, houve o registro de um caso de Raiva animal detectado em um morcego não hematófago (São Paulo, 2020). Em relação a Raiva em seres humanos no estado de São Paulo, durante o mesmo intervalo de tempo, houve uma única ocorrência em 2018, a qual tratava-se de um homem oriundo do estado do Paraná que foi infectado ao ter contato com um morcego durante uma viagem ao município de Ubatuba (Brasil, 2021).

As alterações ambientais provocadas pelos seres humanos tornaram as áreas urbanas em locais propícios para a criação de colônia de morcegos, atrelado com o intenso abandono de felinos, que por instinto predador e de caça aos morcegos, se insere exponencialmente ao ciclo epidemiológico da Raiva como um transmissor exponencial. Acrescido a isto, comumente os felinos são vistos nas ruas em condições de saúde, alimentação e de cuidados inadequados à espécie. Ademais, alia-se o fato de que os humanos consideram os felinos como ser "natural" das ruas, e tolera-o de maneira extremamente próxima num contexto social intradomiciliar. Essas condições sinérgicas propiciam a veiculação de zoonoses como a Raiva (Genaro, 2010; Babonni et al., 2011).

Para a prevenção desse agravo, é recomendado o uso de vacinas de cultivo celular, devido esta apresentar imunização comprovada, maior segurança e menores riscos de reações adversas. Tal vacina é utilizada no Brasil desde 2003, é gratuita e encontra-se disponível em toda rede do Sistema Único de Saúde (SUS) (Who, 2016).

O controle da Raiva humana está associado a diversas condutas de saúde e de educação, abrangendo ações individuais e coletivas, incluindo a vacinação animal, a captura para controle de mamíferos silvestres e a profilaxia pré e pós-exposição para o ser humano, além da vigilância epidemiológica efetiva e adequada (Reichman et al., 2000). Avanços foram obtidos no controle desta zoonose, com uma significativa redução dos casos de Raiva canina transmitida aos seres humanos, principalmente em função das atividades desenvolvidas ao controle da Raiva em cães (Babonni et al., 2011).

Mediante o exposto, o estudo teve como objetivo mapear o padrão da distribuição espacial sobre o conhecimento e práticas de vacinação acerca da zoonose Raiva na população atendida nas Unidades Básicas de Saúde (UBS) do município de Birigui, São Paulo, Brasil.

\section{Metodologia}

Tratou-se de um estudo epidemiológico observacional analítico, não experimental, com delineamento transversal, realizado no período de janeiro a julho de 2019. Localizado no Brasil, situado na região noroeste do estado de São Paulo, o município de Birigui faz parte da região administrativa de Araçatuba, sua população estimada é de 120.692 habitantes, possui o Índice de Desenvolvimento Humano (IDHM) de 0,780, em 2010, obtendo assim um IDHM elevado (IDHM entre 0,700 e 0,799) (IBGE, 2019).

A população de Birigui foi escolhida para o estudo pela amostra acessível para o desenvolvimento da pesquisa devido à proximidade com a UNESP - Universidade Estadual Paulista Júlio de Mesquita Filho - Campus de Araçatuba - Faculdade de Medicina Veterinária - FMVA, e a cooperação de autoridades sanitárias municipais. O município desenvolve o programa de controle de Raiva com a campanha anual de vacinação de cães e gatos, e verificação da circulação viral com remessa de material para o laboratório de Raiva da UNESP - FMVA credenciado pelo Ministério da Saúde (MS). O mesmo dispõe de 10 UBS que oferecem atendimento de saúde por meio do SUS, todas estas, localizadas em perímetro urbano, conforme demonstrado na Figura 1. 
Figura 1 - Localização geográfica de acordo com o IBGE do município de Birigui. Birigui, SP, Brasil, 2019.

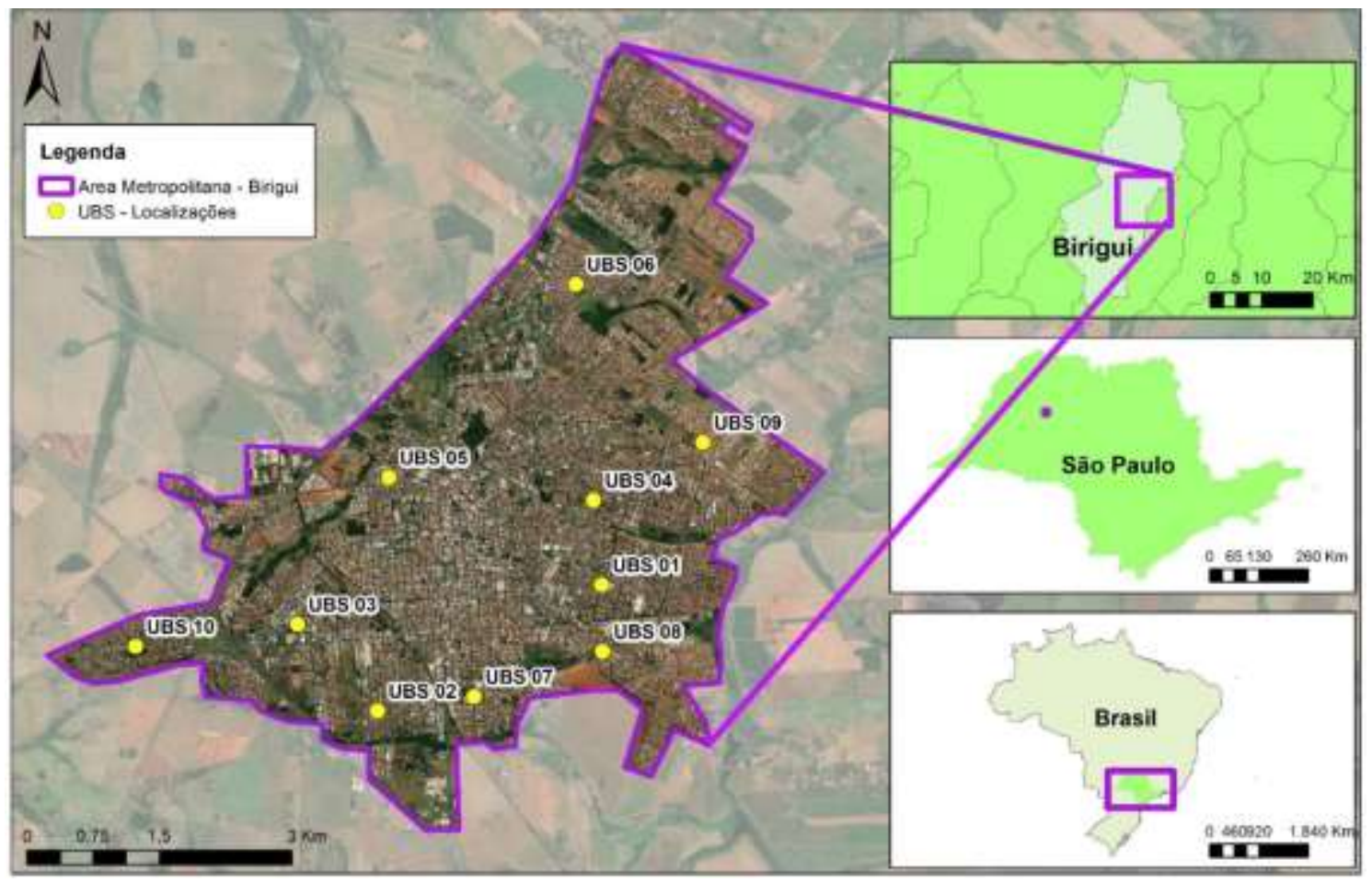

Fonte: Elaborado pelos autores.

Foi desenvolvido um questionário contendo 30 questões sobre as variáveis sociodemográficas dos participantes como sexo, idade, escolaridade, UBS a qual o participante pertencia, bem como questões mais específicas acerca do conhecimento sobre Raiva, como percepção sobre zoonoses, ciclos epidemiológicos da doença, zoonoses mais conhecidas, agente infeccioso e animal transmissor da Raiva, e por fim quanto a periodicidade de vacinação, que foram adaptadas do estudo sobre zoonose Raiva (Tomé et al., 2010), utilizando-se variáveis (sexo, idade, e animal transmissor da Raiva) do estudo (Lovadini et al., 2019) adaptado ao objeto desta investigação.

Para a coleta de dados foram realizadas entrevistas com os participantes do estudo por meio de solicitação verbal, e após aceite, o participante registrava anuência no Termo de Consentimento Livre e Esclarecido (TCLE), as entrevistas foram realizadas nas recepções das próprias UBS e duraram cerca de 10 minutos por participante. Os participantes foram abordados de forma randomizada e de acordo com a disponibilidade do mesmo em responder ao questionário, não havendo estratificação da amostra. Vale ressalvar, que foram selecionados 40 participantes de cada UBS independente do número de famílias abrangentes e da capacidade de atendimento de cada UBS.

O cálculo amostral do estudo foi realizado utilizando um intervalo de confiança de $95 \%$ por meio do programa OpenEpi 3.01. A análise dos dados foi realizada por meio do software Microsoft Office Excel® 2013. Para apresentação das variáveis do estudo, utilizou-se a estatística descritiva, através da transcrição da distribuição relativa (\%) e absoluta (n) dos dados e posteriormente apresentação deles na forma de tabela.

Os critérios de elegibilidade do estudo foram: participantes que se encontravam no local da coleta de dados no momento da pesquisa e que aceitaram responder ao questionário através do TCLE, pessoas maiores de 18 anos de idade, alfabetizadas e moradores do município de Birigui pertencentes e cadastrados nas referidas UBS. Foram excluídos deste estudo todos os participantes que se recusaram a participar da pesquisa por meio de recusa do TCLE, pessoas menores de 18 anos de idade e as analfabetas. 
Para as análises espaciais foi realizada a análise da densidade de pontos, definida como estimador de intensidade de Kernel, que consiste em um método de interpolação exploratória, baseado na definição de áreas circulares de influência em torno de pontos de ocorrências de um fenômeno, gerando uma superfície de densidade para a identificação de áreas vulneráveis (Oliveira et al., 2015).

Para determinar o raio, foi utilizada a ferramenta Incremental Spatial Autocorrelation do software ArcGis 10.5. Essa ferramenta utiliza o Índice de Moran (I) para calcular uma série de distâncias crescentes, medindo a intensidade do cluster para cada distância que é determinada pelo z score. Geralmente, à medida que a distância aumenta, o z score também indica a intensificação do cluster. Em certa distância, no entanto, a pontuação $\mathrm{Z}$ geralmente atinge o pico que reflete a distância onde os processos espaciais que promovem o cluster são mais pronunciados, portanto a distância final a ser utilizada no estudo será a que atingir o pico, isto é, a mais pronunciada, com maior z score e melhor p valor (Oliveira et al., 2015).

Este estudo foi aprovado pelo Comitê de Ética em Pesquisa envolvendo seres humanos da Faculdade de Odontologia de Araçatuba da Universidade Estadual Paulista (FOA-UNESP), sob o protocolo ${ }^{\circ} 3.401 .325$, portanto respeitou os preceitos éticos de cada etapa.

\section{Resultados}

A distribuição sociodemográfica dos participantes da pesquisa, seus conhecimentos e práticas acerca da zoonose Raiva, estão ilustrados na Tabela 1. Cerca de 40 (10\%) participantes estavam cadastrados nas 10 UBS do município, compondo uma amostra de 400 questionários. Quanto ao sexo dos participantes, 297 (74,25\%) pertenciam ao sexo feminino enquanto $103(25,75 \%)$ eram do sexo masculino. Na variável idade, $176(44 \%)$ dos participantes alocavam-se na faixa etária de 31 a 50 anos de idade. No que diz respeito à percepção da população sobre as zoonoses, 197 (49,25\%) responderam que entendem como uma doença transmitida apenas dos animais para os seres humanos.

Ao serem solicitados a citarem duas zoonoses, os participantes apontaram a Raiva e Dengue como zoonose mais conhecidas correspondendo a $222(56 \%)$ dos mesmos. Na pergunta sobre quais eram os ciclos da Raiva, $244(61 \%)$ responderam que existem os ciclos urbano, aéreo, selvagem e rural da zoonose. Ao serem indagados sobre qual era o agente infeccioso da zoonose, $303(75,75 \%)$ assinalaram a alternativa vírus e quanto ao animal transmissor da Raiva 268 (67\%) da população apontaram o cão, gato, morcego e bovino. 
Tabela 1 - Distribuição sociodemográfica sobre a população do estudo e os conhecimentos sobre a Raiva no município de Birigui. Birigui, SP, Brasil, 2019.

\begin{tabular}{|c|c|c|c|}
\hline Variável & Categoria & $\mathbf{n}$ & $\%$ \\
\hline \multirow[t]{4}{*}{ Sexo } & Feminino & 297 & 74,25 \\
\hline & Masculino & 103 & 25,75 \\
\hline & 18 a 30 & 138 & 34,5 \\
\hline & 31 a 50 & 176 & 44 \\
\hline \multirow[t]{2}{*}{ Idade } & 51 a 65 & 54 & 13,50 \\
\hline & 66 ou mais & 32 & 8 \\
\hline \multirow[t]{4}{*}{ Percepção sobre zoonoses } & $\begin{array}{l}\text { Doenças transmitidas dos animais para os seres } \\
\text { humanos e vice-versa }\end{array}$ & 146 & 36,50 \\
\hline & $\begin{array}{c}\text { Doenças transmitidas apenas dos animais para os } \\
\text { seres humanos }\end{array}$ & 197 & 49,25 \\
\hline & Não sei & 57 & 14,25 \\
\hline & Raiva e Dengue & 222 & 56 \\
\hline \multirow[t]{4}{*}{ Zoonoses mais conhecidas } & Leishmaniose e Leptospirose & 170 & 43 \\
\hline & Zika vírus e Brucelose & 2 & 1 \\
\hline & Sarna e Malária & 6 & 2 \\
\hline & Raiva Selvagem & 5 & 1 \\
\hline \multirow[t]{3}{*}{ Ciclos da Raiva } & Raiva urbana, selvagem, aérea e rural & 244 & 61 \\
\hline & Não sei & 134 & 34 \\
\hline & Vírus & 303 & 75,75 \\
\hline \multirow[t]{6}{*}{ Agente Infeccioso da Raiva } & Bactéria & 46 & 11,50 \\
\hline & Fungo & 4 & 0,40 \\
\hline & Não sei & 47 & 11,75 \\
\hline & Cão & 64 & 16 \\
\hline & Gato & 0 & 0 \\
\hline & Morcego & 49 & 12,25 \\
\hline \multirow[t]{4}{*}{ Animal transmissor da Raiva } & Bovino & 4 & 1 \\
\hline & Cão, gato, morcego e bovino & 268 & 67 \\
\hline & Nenhuma das alternativas & 4 & 1 \\
\hline & Não sei & 11 & 2,75 \\
\hline
\end{tabular}

Fonte: Dados da pesquisa.

Na Figura 2, observamos a média da escolaridade dos participantes, segundo o mapa de densidades de Kernel. Ao analisarmos os clusters com maiores índices de escolaridade, as UBS 2, 5 e 7 tinham participantes com níveis mais altos de escolaridades e as com menores índices de escolaridade foram as UBS 4, 3, 8 e 9 respectivamente. 
Figura 2 - Distribuição espacial dos participantes segundo escolaridade de acordo com as UBS, segundo mapa de densidade de Kernel. Birigui, SP, Brasil, 2019.

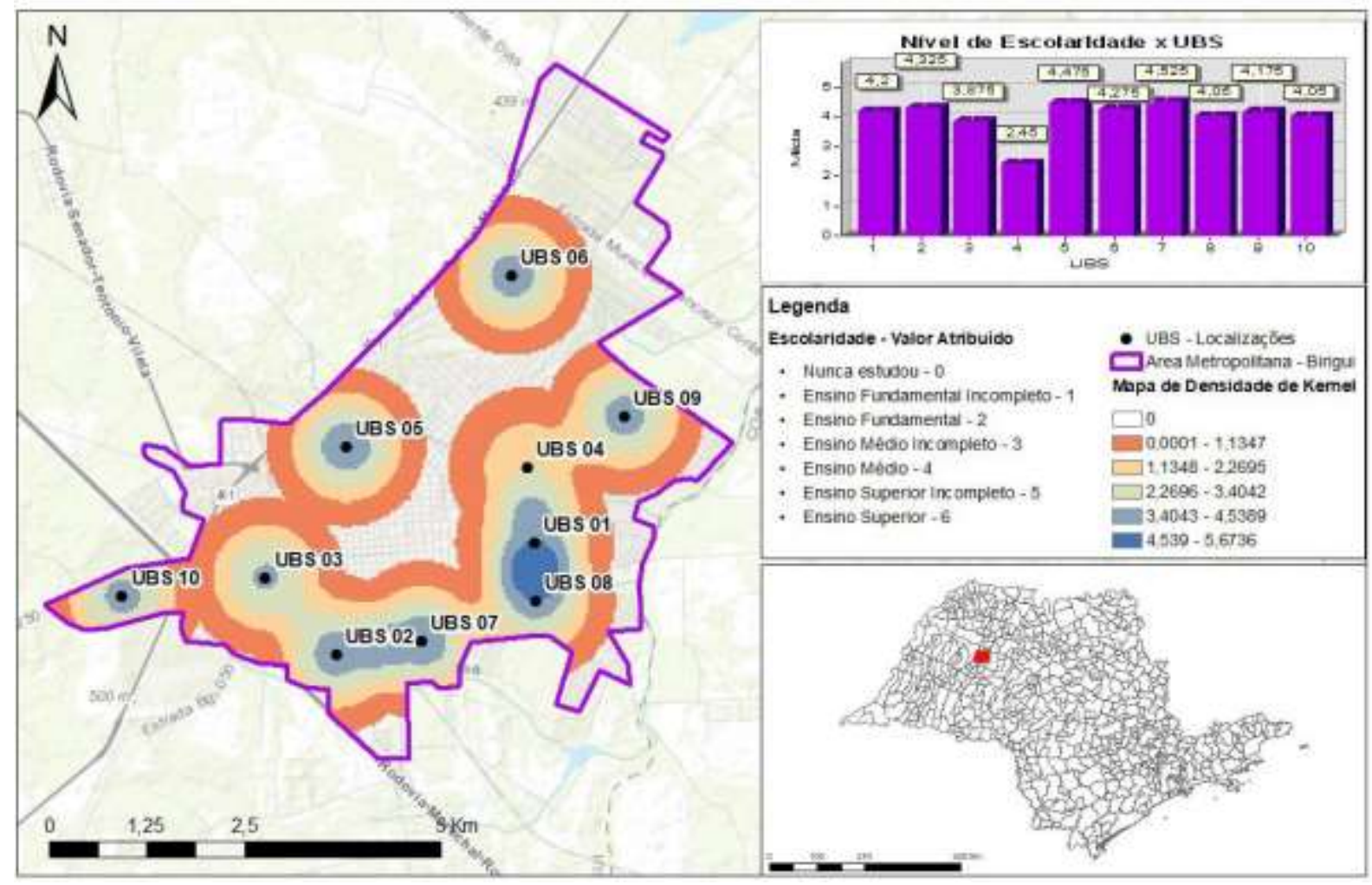

Fonte: Elaborado pelos autores.

Na Figura 3, observamos o número de participantes que responderam corretamente (foi considerado correto a resposta “Cão, gato, morcego e bovino”) sobre os animais transmissores da Raiva segundo o mapa de densidade de Kernel. As UBS de cluster com maior densidade de respostas corretas foi a de número 2, seguida da UBS de número 6 , e encontramos clusters com menores densidades de respostas corretas nas UBS 4, 8 e 10 respectivamente. 
Figura 3 - Distribuição espacial do número de acertos segundo animal transmissor da raiva de acordo com as UBS, segundo mapa de densidade de Kernel. Birigui, SP, Brasil, 2019.

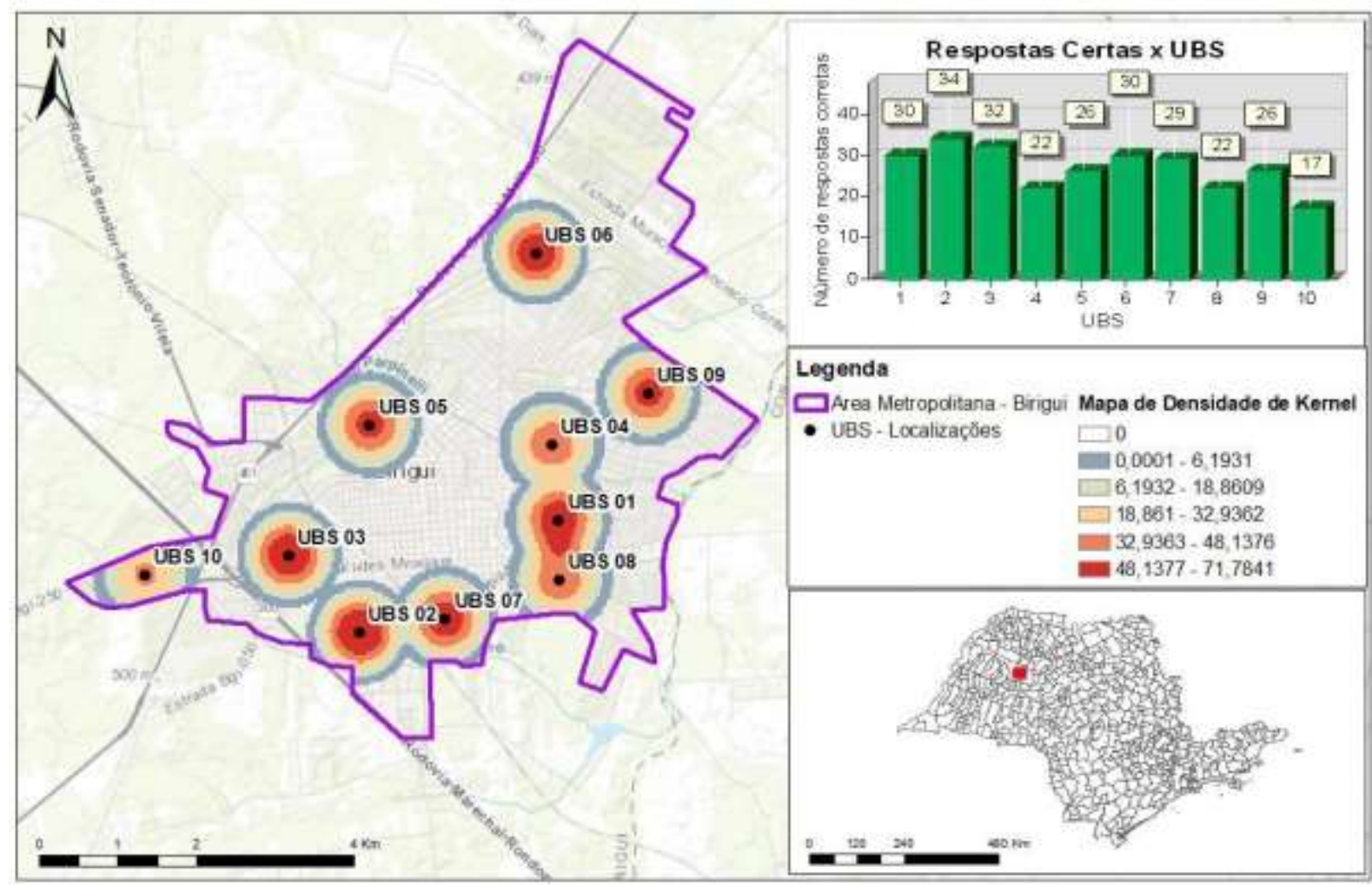

Fonte: Elaborado pelos autores.

Referente à periodicidade de vacinação dos animais de acordo com as UBS, na Figura 4, observa-se que as UBS 8 e 2 respectivamente apresentam densidades pronunciadas no mapa, representando as UBS onde ocorrem corretamente as práticas de vacinação antirrábica, e as de densidades mais baixas, são as de número 9 e 3 respectivamente, representando os locais do município onde as práticas de vacinação ocorrem com menores frequências. 
Figura 4 - Distribuição da periodicidade de vacinação antirrábica anual de acordo com as UBS, segundo Mapa de Densidades de Kernel. Birigui, SP, Brasil, 2019.

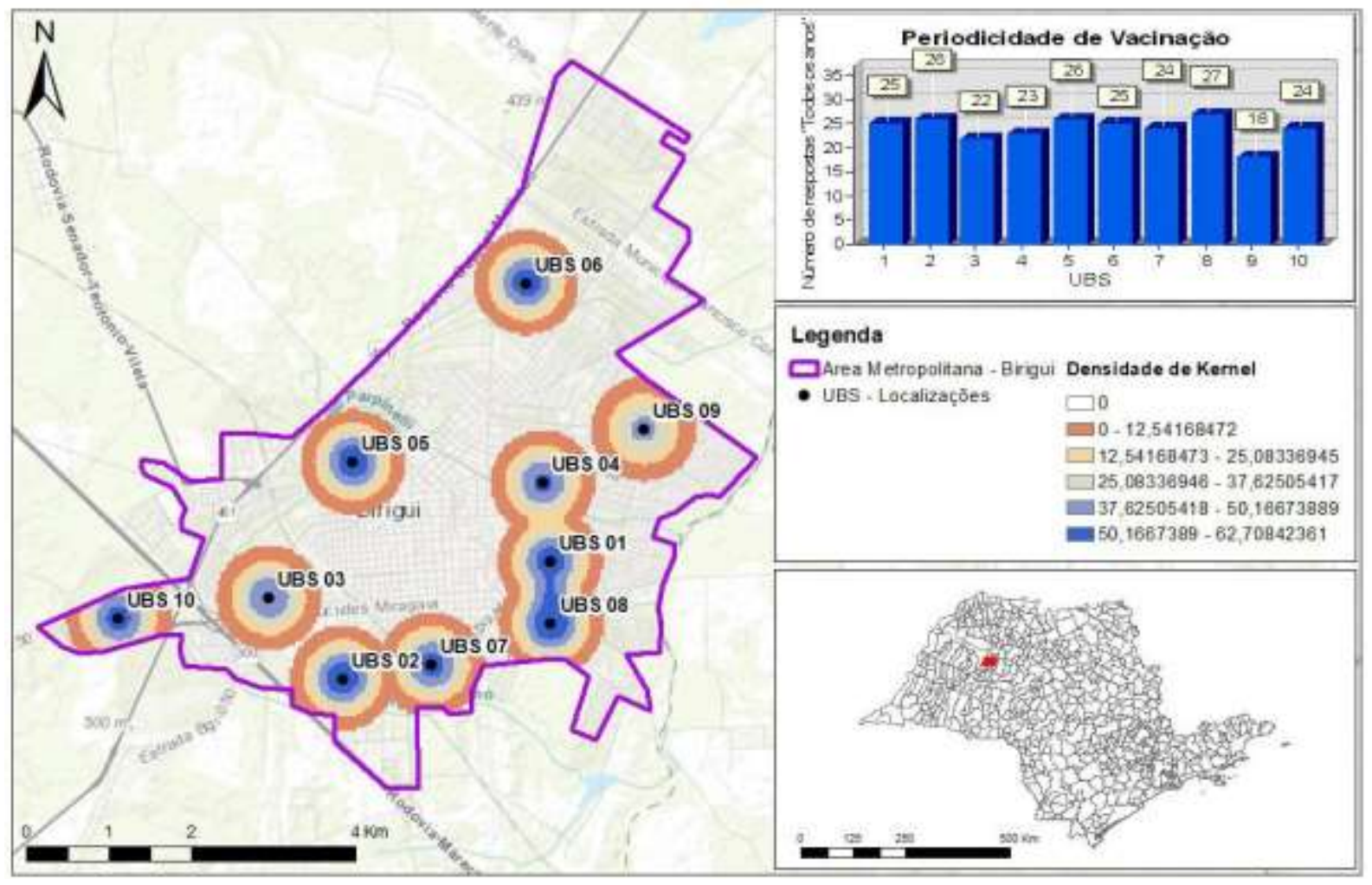

Fonte: Elaborado pelos autores.

\section{Discussão}

Os dados encontrados possibilitaram a aplicação das análises espaciais do conteúdo informativo acerca do conhecimento da população sobre a Raiva, sua transmissão e as práticas preventivas utilizadas pela população estudada.

Os achados de Moran et al., (2015) foram semelhantes aos resultados aqui expostos quando, ao analisarem o conhecimento, atitudes, práticas sobre Raiva e exposição à morcegos em duas comunidades rurais na Guatemala com 270 participantes, pesquisadores relataram que o risco da transmissão por mordedura de morcego raramente era conhecido. Somente $10 \%$ dos 270 interrogados assinalaram os morcegos como os potenciais transmissores, $27 \%$ indicaram os gatos e $14 \%$ não disseram que tomariam alguma ação específica caso fossem mordidos ou arranhados por um morcego.

Segundo Genaro (2010), nas duas últimas décadas a convivência entre humanos e morcegos tem se tornado cada vez mais constante nas áreas urbanas em detrimento de ser um local com abundância de abrigos e alimentos. É fato que, há uma variação no grau de interação humana e comportamento peridoméstico dos morcegos, neste sentido, seus hábitos reclusos tendem a criar situações de exposição incomuns. As espécies insetívoras e fitófagos são mais encontrados dentro das cidades, no entanto, todas as espécies podem transmitir o vírus aos humanos por meio de mordedura, arranhadura ou lambedura. As mordeduras destes, tendem a ser imperceptíveis, isso se deve ao tamanho menor do seu corpo, e principalmente quando comparadas às mordidas e feridas causadas por animais maiores, como os cães.

Apesar do cão ser responsável por $99 \%$ dos casos de transmissão da Raiva aos seres humanos em escala mundial (Carvalho et al., 2018), não se deve subestimar o papel que os felinos e os morcegos possuem nesse ciclo epidemiológico. De acordo com Genaro (2010), o desafio do controle da Raiva urbana nos felinos domésticos, revela o fato de que as espécies de morcegos, são relevantes na veiculação do vírus, e a população felina por sua vez, tende a ter contato próximo com os demais 
animais silvestres, e em detrimento do grau de liberdade destes com seus tutores, pondera-os como um desafio peculiar. Por isso, é recomendado ações de educação em saúde para a população, a fim de incentivar melhores práticas e atitudes frente a tal problemática.

Na América Latina e Caribe, durante o período de 1993 a 2014, houve o registro de 778 casos de óbito de pessoas em decorrência da Raiva, dos quais $49 \%$ foram transmitidos através do contato com cães infectados, os quirópteros eram considerados o segundo maior transmissor da doença, sendo responsáveis por 38\% casos, seguido de espécies agressoras sem registro de espécies com 7\% (WHO, 2020). De acordo com Carvalho et al., (2018), na América Latina, houve uma diminuição dos casos de Raiva em humanos transmitida por cães em 95\% nos últimos 35 anos, sendo que apenas quatro países apresentaram casos (Bolívia, Guatemala, Haiti e República Dominicana) em 2017, sendo esses mesmos países responsáveis por $90 \%$ dos casos de Raiva em caninos domésticos.

Em relação à epidemiologia da Raiva humana no Brasil de 1986 a 2020, é possível observar nos últimos anos uma redução do número de casos registrados desde 2005. Em 2013 houve o último caso de Raiva humana causa pela variável do vírus canino endêmico do território nacional (AgV2) e em 2015 ocorreu o último caso de Raiva transmitida por cães, causado por uma epizootia de Raiva canina na região do Mato Grosso do Sul de uma variante oriunda da Bolívia (AgV1). Assim a partir desse período há um destaque no papel do morcego e do gato como transmissores dessas doenças, pois de 2015 a 2018 foram registrados 20 casos humanos, sendo 17 devido a mordedura de morcegos e dois de felinos, e variável antigênica detectada em ambas as espécies nesse intervalo de tempo (AgV3) típica de morcegos hematófagos Desmodus rotundus, indicando um contato entre esses dois ciclos (Brasil, 2018).

Segundo Kabeta et al., (2015), ao avaliarem o conhecimento, as atitudes e as práticas de vítimas de mordidas de animais que frequentam um centro de saúde antirrábico na cidade de Jimma, Etiópia, no ano de 2014, com 384 indivíduos, observaram que $99 \%$ dos participantes identificaram a mordida de um cão raivoso como a principal maneira das pessoas adquirirem Raiva, $76,3 \%$ indicaram os gatos raivosos, $71,1 \%$ os animais de fazenda e $47,7 \%$ os animais selvagens, ressaltaram também que mais de um terço dos participantes afirmaram que carne e leite de animais raivosos poderiam transmitir também a doença aos seres humanos. Os achados de Fenelon et al., (2013) relataram que dos 550 participantes entrevistados, apenas 29,6\% dos membros da comunidade em Pétionville, Haiti, indicaram os gatos como veículo de transmissão.

De acordo com Hasanov et al., (2018), ao avaliarem o impacto da educação pública em relação à Raiva, observaram que notavelmente menos da metade das 633 pessoas entrevistadas consideraram os gatos domésticos ou sem dono como animais transmissores da Raiva. Esses dados demonstram que houve uma alteração no perfil epidemiológico da transmissão da doença aos humanos, em que o morcego assume o papel de maior importância na vigilância em saúde, uma vez que a Raiva em caninos foi controlada por meio da vacinação.

No que se referem aos dados de cobertura vacinal do município de Birigui, no ano de 2017, a meta de cobertura vacinal dos cães era de 13.296 doses aplicadas, no entanto, apenas 5.909 doses foram realizadas, o que resultou num baixo índice de cobertura vacinal, na qual apenas $44,44 \%$ dos caninos foram imunizados, já entre os felinos, apenas 973 animais foram vacinados (Brasil, 2017). De acordo com os dados do Instituto Pasteur (2018) o número estimado de felinos para o município em questão, em 2018, foi de 2.236, tal fato explicita que nesses a cobertura vacinal foi cerca de 43,51\%. Os dados anteriormente citados vão em contraposição aos achados deste estudo, uma vez que $91,02 \%$ dos participantes referiram realizar vacinação antirrábica anualmente em seus animais domésticos, conforme demonstrado no mapa de densidades de Kernel.

O fato de algumas UBS apresentarem práticas de vacinação com menores frequências que outras, ocorre em detrimento de alguns bairros de abrangência serem localizados e pertencerem às UBS que atendem as periferias do município, o que consequentemente, os tornam mais vulneráveis e implicam em fatores limitadores, a exemplo, os marcadores sociais da diferença (classe social, renda e raça). Essas situações escancaram a problemática retórica de que as mudanças em níveis 
estruturais sofridas pelas cidades têm sido profundas e a urbanização, enquanto dinâmica ambiental e social, pode ser considerada ao mesmo tempo como estruturante e desestruturante (Kabeta et al., 2015).

Ao analisarmos as respostas dos participantes, as UBS 2 e 5 estão entre as que possuem maior nível de escolaridade e melhores práticas de vacinação antirrábica nos animais. Vale ressalvar que a UBS 2 apresentou maior densidade de respostas corretas, por outro lado, as UBS 4 e 8 apresentaram menores densidades de acertos, tanto na variável escolaridade quanto na de respostas corretas, este fato corrobora com os achados de Lovadini et al., (2019), que encontrou associação significativa entre as variáveis citadas, assim reiterando que existe associação entre o grau de escolaridade com o nível de conhecimento, bem como com a periodicidade da vacinação antirrábica.

A baixa escolaridade encontrada neste estudo é reflexo de inúmeros fatores sociais (está relacionada a pessoas em situação de vulnerabilidade social (pessoas que vivem sob condições socioeconômicas precárias sofrendo pela desnutrição, falta de saneamento básico, doenças advindas dessa situação e agravadas pelas desigualdades sociais), econômicos e culturais tendem a potencializar o déficit do conhecimento acerca da enfermidade. Quanto aos achados sobre a escolaridade, observa-se que as UBS não apresentaram uma distribuição homogênea dos conhecimentos e práticas de vacinação sobre a Raiva. Tal distribuição heterogênea se deve aos determinantes econômicos, sociais e culturais distribuídos entre os munícipes (Furlan et al., 2012).

No que tange sobre a relação entre escolaridade e o conhecimento dos participantes sobre a Raiva, notou-se que, os moradores dos bairros centrais detinham mais conhecimentos quando comparados aos residentes dos bairros periféricos. Esse fato explícita à perspectiva que os hábitos em saúde são conexões fundamentais, para que se possa assimilar a propensão do indivíduo ao adotar e manter determinados padrões comportamentais (Queiroz et al., 2009). Outro panorama relevante, são os possíveis fatores limitadores do conhecimento apresentados por parte dos participantes, que pode ser explicado seguindo a proposição de que para adquirir conhecimento, é necessário um conjunto de informações que o indivíduo deve dominar para reger suas práticas em saúde (Moran et al., 2015).

Ao se observarem as respostas dos participantes quanto a percepção sobre zoonoses, nota-se que, 49,25\% dos participantes alegaram que se trata de doenças transmitidas apenas dos animais para os seres humanos. A carência de informação acerca do conceito de zoonose pode ocasionar exposição da população a respeito das medidas preventivas em saúde, levando-as a não realizarem de maneiras adequadas, uma vez que se tratam de doenças que acometem tanto entre o ser humano quanto entre os animais, esses dados corroboram com os achados de Tomé et al., (2010).

Nos estudos de Kabeta et al., (2015), no Azerbaijão em 2017, pesquisadores encontraram entre os 633 entrevistados, que 55,45\% da população analisada realizava vacinação regular, de acordo com a prescrição do médico veterinário. Em países do continente africano os resultados encontrados foram similares, sendo que na Etiópia apenas 41,7\% das pessoas realizavam a vacinação em seus cães, e na Nigéria esse valor seria de 42,4\% da população (Edukugho et al., 2018).

A análise espacial possibilita levar em conta as especificidades dos locais estudados, o que corrobora para uma melhor compreensão de onde aplicar determinadas políticas de saúde de maneira assertiva. Tal ferramenta auxilia na execução das ações de promoção e prevenção em saúde, uma vez que identifica as populações de risco, e sobretudo, permite traçar padrões que poderiam levar ao surto de determinadas doenças, assim evidencia os aspectos epidemiológicos locais, priorizando as medidas de intervenções em saúde adequadas (Khan et al., 2019; Kirby et al., 2017).

Em decorrência dos avanços na geotecnologia, nos é permitido realizar análises espaciais em saúde, que vão além do mapeamento dos eventos, para a reprodução de áreas que permitam análise e reflexão, com a inserção de dados históricos, geográficos, ambientais e socioeconômicos, de maneira intrínseca e extrínseca, implicados no surgimento da doença ou de agravos em espaços temporais definidos (Kirby et al., 2017). 
As limitações do estudo decorrem da falácia ecológica, em que a relação entre o fator de exposição e o evento pode não ocorrer no nível individual. Esse viés pode ocorrer quando se chega a uma conclusão errônea sobre uma associação encontrada entre variáveis no nível agregado que não representa necessariamente uma associação no nível individual. Também vale a pena mencionar o uso de fontes de dados secundários que podem levar a dados incompletos ou erros de digitação.

Esperamos que estes resultados possam estimular e direcionar as autoridades locais para uma tomada de decisão, no intuito de melhorar a prevenção e o controle da Raiva em cães, gatos, morcegos e em seres humanos por meio de programas educacionais direcionados à comunidade.

\section{Conclusão}

Houve um padrão espacial entre as populações atendidas pelas UBS analisadas, onde se constatou que as que possuíam melhores práticas de prevenção sobre a zoonose se mantiveram também com os maiores índices de escolaridade e acertos quanto aos aspectos epidemiológicos da Raiva. Por outro lado, as UBS que possuíam menores números de acertos, se mantiveram no grupo das populações mais vulneráveis no estudo sob as demais variáveis analisadas.

A análise espacial combinada a fontes de informação contribuiu em uma avaliação mais ampla do perfil do conhecimento dos munícipes sobre a Raiva, identificando os locais onde a população demonstrou conhecimento mais acentuado sobre a zoonose, bem como, os locais mais vulneráveis neste viés. Tais ferramentas, são fundamentais para a consolidação de estratégias em campanhas de educação em saúde para o controle e prevenção da Raiva urbana. Diante disso, sugerimos que outros estudos sejam conduzidos sob a perspectiva de análises espaciais quanto cobertura vacinal antirrábica.

\section{Agradecimentos}

O presente trabalho foi realizado com apoio da Coordenação de Aperfeiçoamento de Pessoal de Nível Superior Brasil (CAPES) - Código de Financiamento 001.

Agradecemos as autoridades sanitárias do município de Birigui que concedeu as autorizações para a realização da pesquisa.

\section{Referências}

Abela-Ridder, B., Martin, S., Gongal, G., \& Engels, D. (2016). Rabies vaccine stockpile: fixing the supply chain. Bulletin of the World Health Organization, 94(9), 635.

Babboni, S. D., \& Modolo, J. R. (2011). Raiva: origem, importância e aspectos históricos. UNOPAR Científica. Ciências Biológicas e da Saúde, $349-356$.

de Lima Lovadini, V., Lorena, L. L. G., da Silva Lacerda, J., \& Marinho, M. (2019). Percepção e práticas da população atendida nos serviços primários de saúde sobre a Raiva. Revista Enfermagem Atual In Derme, 90(28).

de Oliveira, B. C. M., \& Gomes, D. E. (2019). Raiva-Uma Atualização Sobre A Doença. Revista Científica, 1(1).

Edukugho, A. A., Umoh, J. U., Diem, M., Ajani, O., Uba, B., Okeke, L., \& Nguku, P. (2018). Knowledge, attitudes and practices towards rabies prevention among residents of Abuja municipal area council, Federal Capital Territory, Nigeria. Pan African Medical Journal, 31(1).

Fenelon, N., Dely, P., Katz, M. A., Schaad, N. D., Dismer, A., Moran, D., \& Wallace, R. M. (2017). Knowledge, attitudes and practices regarding rabies risk in community members and healthcare professionals: Pétionville, Haiti, 2013. Epidemiology \& Infection, 145(8), 1624-1634.

Furlan, M. C. R., Oliveira, S. P. D., \& Marcon, S. S. (2012). Factors associated with nonadherence of tuberculosis treatment in the state of Paraná. Acta Paulista de Enfermagem, 25, 108-114.

Genaro, G. (2010). Gato doméstico: futuro desafio para controle da raiva em áreas urbanas? Pesquisa Veterinária Brasileira, $30,186-189$.

Gomes, M. N., Monteiro, A. M. V., \& Escada, M. I. S. (2011). Raiva bovina segundo os mosaicos de uso e cobertura da terra no estado de São Paulo entre 1992 e 2003. Arquivo Brasileiro de Medicina Veterinária e Zootecnia, 63, 287-295.

Hasanov, E., Zeynalova, S., Geleishvili, M., Maes, E., Tongren, E., Marshall, E., \& Horton, D. L. (2018). Assessing the impact of public education on a preventable zoonotic disease: rabies. Epidemiology \& Infection, 146(2), 227-235. 
Instituto Brasileiro de Geografia e Estatística (IBGE). Estimativas da população residente com data de referência $1^{\circ}$ de julho de 2019. [Internet] Instituto Brasileiro de Geografia e Estatística; 2019. Disponível em: https://cidades.ibge.gov.br/brasil/sp/birigui/panorama.

Ito, M., Arai, Y. T., Itou, T., Sakai, T., Ito, F. H., Takasaki, T., \& Kurane, I. (2001). Genetic characterization and geographic distribution of rabies virus isolates in Brazil: identification of two reservoirs, dogs and vampire bats. Virology, 284(2), 214-222.

Kabeta, T., Deresa, B., Tigre, W., Ward, M. P., \& Mor, S. M. (2015). Knowledge, attitudes and practices of animal bite victims attending an anti-rabies health center in Jimma Town, Ethiopia. PLoS neglected tropical diseases, 9(6), e0003867.

Khan, A., Ayaz, R., Mehtab, A., Naz, K., Haider, W., Gondal, M. A., \& Ahmed, H. (2019). Knowledge, attitude \& practices (KAPs) regarding rabies endemicity among the community members, Pakistan. Acta tropica, 200, 105156.

Kirby, R. S., Delmelle, E., \& Eberth, J. M. (2017). Advances in spatial epidemiology and geographic information systems. Annals of epidemiology, 27(1), 1-9.

Ministério da Saúde (BR). Campanha nacional de vacinação anti-rábica, 2017: doses aplicadas e cobertura vacinal dos municípios do estado São Paulo. In: Sistema de Informação do Programa Nacional de Imunizações (SI-PNI). Ministério da Saúde: Sistema de Informação do Programa Nacional de Imunização; 2013

Ministério da Saúde (BR). Raiva https://www.gov.br/saude/pt-br/assuntos/saude-de-a-a-z-1/r/raiva.

Moran, D., Juliao, P., Alvarez, D., Lindblade, K. A., Ellison, J. A., Gilbert, A. T., \& Recuenco, S. (2015). Knowledge, attitudes and practices regarding rabies and exposure to bats in two rural communities in Guatemala. BMC research notes, 8(1), 1-7.

Oliveira, U., Brescovit, A. D., \& Santos, A. J. (2015). Delimiting areas of endemism through kernel interpolation. PloS one, 10(1), e0116673.

Pan American Health Organization (PAHO). Rabies persists in only four countries of Latin America and the Caribbean. https://www.paho.org/en/news/21-92018-rabies-persists-only-four-countries-latin-america-and-caribbean.

Queiroz, L. H., Carvalho, C. D., Buso, D. S., Ferrari, C. I. D. L., \& Pedro, W. A. (2009). Perfil epidemiológico da raiva na região Noroeste do Estado de São Paulo no período de 1993 a 2007. Revista da Sociedade Brasileira de Medicina Tropical, 42, 9-14.

Reichmann, M. D. L. A. B., Pinto, H. D. B. F., Arantes, M. B., Santos, M. B. D. S., Viaro, O., \& Nunes, V. D. F. P. (2000). Educação e promoção da saúde no Programa de Controle da Raiva. In Educação e promoção da saúde no Programa de Controle da Raiva (pp. 28-28).

Rysava, K., Mancero, T., Caldas, E., de Carvalho, M. F., Castro, A. P., Gutiérrez, V., \& Hampson, K. (2020). Towards the elimination of dog-mediated rabies: development and application of an evidence-based management tool. BMC infectious diseases, 20(1), 1-14.

São Paulo. Instituto Pasteur. Secretária da Estadual de Saúde. Programa Estadual de Controle de Raiva: número de amostras positivas para raiva por mês, município e espécie animal. Número de Amostras Positivas para Raiva por mês, município e espécie animal. 2020. http://www.saude.sp.gov.br/resources/instituto-pasteur/pdf/dados-estatisticos/amostraspositivasderaivaemanimalpormesnoestadodesaopaulo2020.pdf.

Secretária da Estadual de Saúde do Estado de São Paulo (BR). Populações de cães e gatos 2018. In: Instituto Pasteur; 2018.

Tome, R. O., Langoni, H., Peruca, L. C. B., \& Babboni, S. D. (2010). Avaliação do conhecimento sobre algumas zoonoses com proprietários de cães da área urbana do Município de Botucatu-SP. Journal of Health Sciences, 12(3).

Vos, A., Nunan, C., Bolles, D., Müller, T., Fooks, A. R., Tordo, N., \& Baer, G. M. (2011). The occurrence of rabies in pre-Columbian Central America: an historical search. Epidemiology \& Infection, 139(10), 1445-1452.

World Health Organization. (2016). Generic framework for control, elimination and eradication of neglected tropical diseases (No. WHO/HTM/NTD/2016.6). World Health Organization.

World Health Organization. (2019). Driving progress towards rabies elimination: new WHO recommendations on human rabies immunization and results of Gavi's learning agenda on rabies and 2nd international meeting of the Pan-African Rabies Control Network (PARACON): meeting report, 12-14 September 2018, Johannesburg, South Africa (No. WHO/CDS/NTD/NZD/2019.02). 